\title{
Concerning Baraka's Paradoxes
}

\author{
Victor Peterson II
}

Abstract: In what's to follow, we explore the implications of the Baraka Paradox, "hell is definitions." These issues come to light with regard to the logic of identity. Navigating the frames of reference organizing the states of affairs one inhabits, the functional content of identity assertions actively construct those states with many consequences to bear in mind for those utilizing those terms.

Ø. 1965. Amiri Baraka, then LeRoi Jones, states in The System of Dante's Hell that, "Hell is your definitions." 1 Below, we will explore the functional content of this assertion. In so doing, we will illustrate the arbitrary stipulation of identity assertions that, when put to use, are employed towards necessary and particular effect. As such, use implicates the one utilizing those markers in the construction of the contexts in which what was asserted becomes appropriate because used to construct that very context and it's successors. As names can point to multiple entities, and multiple entities can be known under the same name, nomination seems not to refer to "things" in the world. Rather, their content seem a function indexed to an appropriate context of assertion; known as such because a successor of an initial context of nomination. This realization leads to many interesting inconsistencies with regard to reference and identity.

1. The first issue to take up is that of identity as the interchangeability of individuals marked by roles within the formulae that make up how one's state of affairs is organized. To make a start, a few principles must be understood regarding the validity of statements made and the consistency of the systems these statements construct. A statement of "one thing AND another thing" being a part of this context is valid if both objects are in this same context; a statement that says that "one thing OR another thing" is valid if either or both are members of this context; and "IF one THEN the other" is a valid statement only when the antecedent object is either of the context or not and the consequent is of the context. The antecedent represents a sufficient but not necessary condition for the consequent. Thus IF/THEN is only invalid when the antecedent obtains and the consequent does not. If within a system of statements one finds that one statement is valid of something and another is invalid of the same thing, then that system is inconsistent. A statement is valid in saying that something is not of this context and it is indeed a member of another context (NEGATION). Negation, i.e. NOT-"something", is arithmetically represented as a negative value, which is to say, i.e. "X-(something)." With this understood, let us continue.

Categorization, for now, can be said to work by the formula:

\section{[1] IF A, THEN B, given A}

where "given" indicates modus ponens. More often than not, if A is made identical with some other thing, this statement is rendered false. Crudely put, [1] can be made to say of an individual: IF 'dark-skinned' THEN 'Black' given 'dark-skinned'. It is rather simple to construe an identity statement substituting 'darkskinned' with some other presumed marker of Black identity that substitution, however, makes the rule above false.

Proof. If $x$ is an element of a class A and A is an element of the class B, if A and B are not identical, then $x$ and NOT- $x$ are elements of B. As both $x$ and NOT $-x$ are in B, their union represents the set of elements that are not members of themselves, the null class. Since the null is in B and A is in B, then null is in A, 
when A is without no members. As the zero of A, the zero being a class/category with as yet no members, this point represents the functional content of its assertion, the application of which constructs, i.e. populates A. If being an element of $\mathrm{A}$ is to have some property, then the above is valid for there is no one element as yet that does not have that property; the functional content of A is yet to be applied. Thus, if $f_{a}=0$, then IF $f_{a}$ THEN A. If A then B given A, then $f_{a}(\mathrm{x})$ is in B given $f_{a}$. As such $f(f(\mathrm{a}))=\mathrm{B}$ and when $\mathrm{a}=0$, then we get the functional content of B. In this way, if B is affirmed in "IF A THEN B", B is either vacuous or contradictory for $f_{\mathrm{a}}=\mathrm{B}$ which is invalid. So to affirm $\mathrm{B}$ is merely to affirm $f_{b}$ at 0 . We conclude that null is the basis for constructing what is asserted,2 and as having only functional content, it is not empty and is a part of and yet remains in excess to, i.e. apart from, every set constructed. Here, $f_{a}$ constructs the conditions in which what is classified as A or B obtains. 3

We wish to get to the formal or underlying structure to why the system of categorization expressed is inconsistent. Thus, B is necessary if and only if for all A, IF A THEN B. Below, we shall see how categorization by virtue of identity stipulation can return vacuous results, implying their necessity to the system constructed, not to the inhabitants of the state their employment seeks to organize.

Take two names of the same individual: the poet LeRoi Jones, later known as Amiri Baraka. We want to say that LeRoi is identical with Amiri such that: LeRoi=Amiri. According to [1] and adopting the same form, we state:

[1.1] IF LeRoi, THEN Amiri, given LeRoi.

We introduce the symbolism of identity, =, as IS. Supposing this is valid, we state of LeRoi:

[1.12] LeRoi IS a Beat Poet; or LeRoi=Beat

Substituting 'Beat' for 'LeRoi', we derive the false statement:

[1.13] IF a Beat, THEN Amiri given a Beat

for Amiri Baraka was not a Beat Poet but a Black Arts Movement poet although a name of the same individual.

This example was chosen for two reasons: i) it shows the inconsistency with considering only the intersection of categories themselves as exhaustive or capturing all members of a state of affairs that fit certain criteria; ii) it illustrates the problem that arises when identity becomes the sole indicator of one's membership within a state of affairs. The latter statement is an issue of reference between label and thing. Formally, considering names as predicates with functional content, we wish to say:

$[1.2](\mathrm{L}=\mathrm{A})$ is true

Thus, for some $\mathrm{X}$ or stating that, "there is an X," L and A are identical. Therefore, we only require one and seem to get both. So we state:

[1.21] L AND $X$ is also true, thus LX

However, LX does not equal $\mathrm{L}$ for "L AND X" is not just $\mathrm{L}$ or just $\mathrm{X}$. $\mathrm{L}$ and $\mathrm{X}$ are members of the same context in order for that individual named "LX" to be LX in those conditions. For the statement "LX" being a member of that context at all or, even if a member, being the individual that is " $\mathrm{L}$ " is contingent. If $\mathrm{L}=\mathrm{X}$, exchange $\mathrm{L}$ for $\mathrm{X}$ or $\mathrm{X}$ for $\mathrm{L}$, we lose either $\mathrm{L}, \mathrm{X}$ for the sake of one. $\mathrm{LX}$ and $\mathrm{L}$ can name separate individuals in the same context. Therefore, 
save by extra-contextual stipulation. If "IF X THEN, IF L THEN A" presents a modal expression of A, then,

\section{[1.23] IF X THEN, IF L THEN A, given L OR X}

Since it is undecidable whether $\mathrm{X}$ or $\mathrm{L}$ gives rise to $\mathrm{A}$, we can say:

[1.24] Given LX, possibly NOT-A

for possibly-(IF LX THEN A)= "(IF LX THEN A) OR NOT-(IF LX THEN A)"; the right being equivalent by way of our formation rules to "(NOT-LX OR A) OR (LX AND NOT-A)". Taking this further, we can state:

\section{[1.25] IF A THEN NOT-A}

given the contingency of LX as the consequence of [1.24], which results in (A AND NOT-A). We see that NOT-A is imposed wherever A is asserted. Consider "IF NOT-LX OR A, THEN LX AND NOT-A" we get "NOT-A OR, LX AND NOT-A" which is to say: "IF NOT-LX THEN, IF A THEN, IF LX THEN NOT-A". So, asserting the conditional "IF LX THEN, IF A THEN LX" we can say "IF A THEN NOTA" represents contingent conditions.

From this we could infer: IF possibly-NOT-NOT-A, THEN NOT-A. We then arrive at:

[1.26] necessarily NOT-A, is equivalent to, NOT-possibly-NOT-NOT-A

making it necessarily $(\mathrm{L} \neq \mathrm{A})$. Quantification into opaque contexts is proven false by identity rules due to the expression of modality implicit in making those stipulations.4

2. Consider another example. Take two identifiers for the same individual, ' $a$ ' and ' $b$ '. Let's also suppose that a label is essential to an individual, a point necessary to quantify the inhabitants of some state of affairs. Finally, let's assume the limits of that context are indeterminate. From here we state:

[2] $a=b$

From [2], we can abstract a functions that makes identity within this system a predicate, describing an individual's existence by virtue of their significance within that system. Abstraction allows us to assume that the content of an assertion is functional. As a relation between names obtains in the context constructed by the employment of those assertions, their use are appropriate therein due to their prior involvement in constructing the contexts of their assertion. In other words, we formalize the function over an individual's operation under that name, e.g. its "communal" role.6 So,

$[2.1] f_{\mathrm{x}}(x=a) b$

[2.1] states that of $b$ there is a functional predicate which makes it identical, i.e. interchangeable, with another individual $a$, indicating their membership within the same category. Substitute $b$ for $\mathrm{x}$, we supposedly obtain $a=b$ as output. Thus, $a(b)$, where $a$ on its own is not defined by some other object but by its functional content, constructing the contexts in which its assertion, i.e. pairing with $b$, is 
appropriate. $b$, then, becomes an input of this function which sets its role, because substitutable with, $x$. Let us call this an Id function. One that overdetermines $b$ 's relation, i.e. sets the function of $b$, within this state of affairs. So we have:

$[2.2] \mathbf{I d}(f \mathrm{x}): x=a(b)$

" $(x=a)$ " is now a predicate as the Id function of the function $f_{\mathrm{x}}$ expresses the concept or system itself, not the objects in its domain. The function of that operation expresses a system of categories or a particular arrangement of predicates, for example Race. As such, $f_{\mathrm{x}}(x=a) \mathrm{b}$ or $\mathbf{I d}(b)$ in accordance with [2.12], predicates or labels $a$ over $b$ such that:

$[2.21] \mathbf{I d}(a(b))$

[2.21] is an identity statement whose value is only obtained within this system of categorization.

However, to retain a relation between the two, we want for $a$ to at least be identical with $a$, i.e. $a=a$ :

[2.22] $\mathbf{I d}(a):(a=a)$

But, as $b$ can also be stated as not- $a$, substituting not- $a$ instead of $b$ in accordance with [2], we obtain:

[2.23] Id(not- $a):($ not $-a=a)$

which is false. Categorization via the operation of the Id function leads to inconsistent outcomes without extra-operational stipulation.

An Id function that states that $a$ overdetermines or sets the function of $b$ reveals that, e.g. racial identification, is an empty predicate which may or may not be fulfilled with an individual member of the domain of the state dependent upon the context in which that individual participates. If that individual does not participate within that system of categorization, this does not mean that that individual does not exist, but affirms that it does, just not in this particular world arrangement.7

3. Consider the difference between the following: 'may', indicative of modality; 'not' indicating negation, such that:

[3] . . may not---

[3.1] . . not may---

Semantically, it is acceptable to state [3]. However, [3.1] is invalid. It is nonsensical for negation to precede modality. There is a not so obvious syntactic reason that further explains this inconsistency. We can uncover this discrepancy if we consider existence as a predicate.

Consider again the names, 'LeRoi Jones' and 'Amiri Baraka.' We choose these two in order to consider the consequences of a proposition made in, The System of Dante's Hell, in which he questions whether or not one can exist as an operation or must so only by virtue of a defined role. His conclusion, "Hell is definitions."

Let LeRoi Jones ( $\mathrm{J}$ ) and Amiri Baraka (B) be different names for the same subject. Each nominal function expresses a different objective use within our reference frame. $\mathrm{L} \neq \mathrm{B}$ as $\mathrm{L}=\mathrm{L}$ and $\mathrm{B}=\mathrm{B}$. We can conclude that these names are different with regard to their significance within the form of life their use constructs in the same state of affairs. That state is organized by the reference frames of which those names are a 
part. The subject, expressed by the utilization of that function-expressing its self through the operation of that nominal predicate - remains the same. Thus, a subject X may obtain a relation between $\mathrm{J}$ and B as follows.

First, let's get clear on some formation rules. Consider two assertions X,Y. We can define their functional content by naming the function of a predicate such that what proceeds it becomes a variable that can be exchanged for what is available to us. In other words, we have an identifier and an expression such that $\mathbf{I d}\left(\mathrm{E}_{1}\right)$ and, given $\mathrm{E}_{2}$, we say $\mathrm{E}_{1}\left(\mathbf{I d}=\mathrm{E}_{2}\right)$. The result in parenthesis is held in that domain until all E's are Id'd, then we move to the next domain. If, in the domain in which that function operates, what is required is not present, no output is expressed. We engender an order of operations with which we can test the validity of expressions. Names are a great candidate for this test as nomination does not necessarily pick out a "thing" per se; rather, they index assertions to the appropriate conditions in which they are expressed. Hence one can talk about something without its being present in such a way that others understand what you're talking about.

Given two predicate functions X,Y:

\section{[3.2] $\mathrm{X}(\mathrm{Y})$ given $\mathrm{X}$}

is valid for $\mathrm{Y}$ is a variable in the domain of $\mathrm{X}$, and when given an $\mathrm{X}$ to be exchanged for that variable in the domain predicated as " $\mathrm{X}$ ", the result satisfies that original predicate such that $\mathrm{X}=\mathrm{X}$ for $\mathrm{Y}$ is now $\mathrm{X}$ in that domain. Thus,

\section{[3.22] X(Y) given $\mathrm{Y}$}

is invalid. We can go on to say that given a proposition $p$ that takes a value as defined above and two assertions $\mathrm{A}, \mathrm{B}$, we read $p \mathrm{AB}$ as "IF $p$ THEN A, else B".

So, going back to our names J and B we show,

$$
[3.23] \mathrm{X}(\mathrm{JB})
$$

Given $\mathrm{X}, \mathrm{J}=\mathrm{X}, \mathrm{B}=\mathrm{X}$, and therefore $\mathrm{X}=\mathrm{X}$. Given $\mathrm{J}$ or $\mathrm{B}$ alone returns invalid results. Thus, $\mathrm{J}$ and $\mathrm{B}$ are $\mathrm{X}$ related in this context, but are not interchangeable.

We know J,B are not the same but are a function of the same object. Consider the following,

$$
[3.24] \mathrm{JB}(\mathrm{X}(\mathrm{J})(\mathrm{B}))
$$

such that given $\mathrm{X}$ of $\mathrm{J}$ or $\mathrm{B}$, then $\mathrm{JB}$ of $\mathrm{X}$. So, if given an $\mathrm{X}$, the formula computes, i.e. reduces for $\mathrm{B}=\mathrm{X}$, $\mathrm{J}=\mathrm{X}$, therefore the expression is $\mathrm{JB}$ of $\mathrm{X}$. If given $\mathrm{J}$ alone, $\mathrm{J}=\mathrm{J}$ but not $\mathrm{X}$ nor $\mathrm{B}$. The operation holds in that domain until whatever else is required as input becomes available, in whatever successive order. If what is required as input is not available, the function produces no output as an identifiable solution. We arrive at this reading because in the domain in which $\mathrm{X}$ is free, $\mathrm{X}$ can be paired with $\mathrm{J}$ or $\mathrm{B}$. So where $\mathrm{J}$ or $\mathrm{B}$, if given $\mathrm{X}$ of $\mathrm{J}, \mathrm{X}$ of $\mathrm{B}$, we can surmise that if $\mathrm{JB}$ of $\mathrm{X}, \mathrm{XJ}$ or XB. A property that holds of entities together does not necessarily mean that that property holds for each individually.

When considering an expression of X, that expression's functional content constructs the contexts in which $\mathrm{J}$ or B is a member. From this, we can illustrate successor-contexts that refer to these original conditions as the set $\{\mathrm{X}: \mathrm{J}, \mathrm{B}\}$. In this way the disjoint union of $\mathrm{J}, \mathrm{B}$ at $x_{0}=f_{\mathrm{x}}$, i.e. the functional content constructing the sets in which $\mathrm{X}$ is implicated due to the application of its functional content, makes it 
such that $\mathrm{J}, \mathrm{B}$ are $\mathrm{X}$ related by that function. The subsequent contexts in which either $\mathrm{J}$ or $\mathrm{B}$ are asserted are such that in the former we have $\mathrm{J}$ indexed to its context by $\mathrm{x} 0$, in which $\mathrm{J}$ is asserted as the successor context, thus $\mathbf{J}_{1}$, and $\mathrm{B}$ is not. Although $\mathrm{B}$ does not obtain there, it's function is present at its zero, i.e. as yet to be applied. Thus, the successor-set contains $\left\{\mathrm{X}_{0}\left\{\mathrm{X}_{1}, \mathrm{~B}_{0}, \mathrm{~J}_{1}\right\}\right\}$. The converse holds as well so that the possible articulations of $X$ is in the set $\left\{X_{0}\left\{B_{0}, J_{1}\right\}\left\{B_{1}, J_{0}\right\}\right\}$ which is equivalent to $\{X\{J, B\}\}$.

[3.25] Proof. $\{\{J, X\} B\}=\{J\{X, B\}\}$ is equivalent to $\{0\{0\}\}=\{0,\{1\}\}$ so $J(X)=1$ and $B=B=0$. Same for the latter. 0 n is just 0 . An expression of $X$ is had by taking " 0 " as a set and the pair of that constant with another 0 as a set of that set and label. Thus, $X$ as constant connects functions as yet applied to the one asserted and itself. E.g. $\{0,0,0\}=\{0\}$ is the context in which $X=X$ thus $\{0\{0\} 0\}$ is the same as $\{0\{0\}\}$ the subset is where we assert $X$ and label it for $\{0\{0,1\}\}$ is the same for 0 and 1 is just 1 .

So, "J"="B" for wherever the name J or B are free, i.e. affixed to no one thing and considered alone; but when $\mathrm{J}$ and $\mathrm{B}$ are bound within a particular domain qua reference frame, they are not the same for each set has the same content but arranged, expressed, in different ways. According to Peano's axioms, $f_{\mathrm{xj} b}=0$ where " $\mathrm{J} "=f_{\mathrm{j}}$ and " $\mathrm{B} "=f_{\mathrm{b}}$. We know the appropriate assertion of each insofar as they do not apply, i.e. are outside the scope of the context as the subset in which name and object are paired; thus $\mathrm{J}$ and $\mathrm{B}$ are not the same yet $\mathrm{J}(0)=\mathrm{B}(0)$ thus $\mathrm{X}=\mathrm{X}$ for $\mathrm{X}$ is constant in both constructions so $f(0)=\mathrm{c}=\mathrm{X}$. In this way a successor from $\mathrm{X}$ labeling $\mathrm{X}$ in the context constructed is $f(f(0))=1$ and each label having the same content $1=1$.

When $\mathrm{X}$ is in the context of $\mathrm{J}$ or B, X's name is what's bound as the set which becomes the context. Thus, assertion of X's label is context dependent upon the function applied over the function indexing it to the context under consideration. In other words, names context with respect to constant in both contexts, $\mathrm{X}$.

By separation, the modalities in which the subject $X$ can be expressed will always be in excess of the identities available. For example, the set of even numbers is made of natural numbers but is itself not a natural number.

Following the implications of negating X before its expressed modality through J and/or B (cf. [3]-[3.1]), we see from [3.2]-[3.25] that issues arise once existence is made a predicate of $\mathrm{J}$ and $\mathrm{B}$. If we abstract from (JB) an existential function $f$ qua predicate $\mathrm{E}$, where $f=\mathrm{E}$, we obtain the formula:

\section{[3.3] $\mathrm{E}(\mathrm{X}(\mathrm{J}=\mathrm{X})(\mathrm{B}=\mathrm{X}))$}

such that $\mathrm{J}$ or B become inputs to test existential predication. With $\mathrm{J}$ and $\mathrm{B}$ equal to the same, "J"="B" for $\mathrm{X}$. We can abstract a function,

\section{[3.31], $\mathrm{E}(\mathrm{X}(\mathrm{X}=\mathrm{J}))$}

making existence a predicate of $\mathbf{J}$ and test the same inputs.

$$
[3.32] \mathrm{E}(\mathrm{X}(\mathrm{X}=\mathrm{J}))(\mathrm{J}) \quad[3.33] \mathrm{E}(\mathrm{X}(\mathrm{X}=\mathrm{J}))(\mathrm{B}) \quad[3.34] \mathrm{E}(\mathrm{X}(\mathrm{X}=\mathrm{B}))(\mathrm{J}) \quad[3.35] \mathrm{E}(\mathrm{X}(\mathrm{X}=\mathrm{B}))(\mathrm{B})
$$

$(\mathrm{J} J)$ just is $\mathrm{J}$ because $\mathrm{J}=\mathrm{J}$. By [3.23], $\mathrm{X}(\mathrm{JB})$ represents the function of the symbolism of identity between names. However, here the names are the individual objects that obtain the existence predicate, whereas before $\mathrm{X}$ was that individual where $\mathrm{J}$ and $\mathrm{B}$ were names of $\mathrm{X}$. From this we derive a contradiction. This should be of no surprise for the zero of a function is the function itself. SO the content of $\mathrm{E}$ is $e 0=f$ where $f(e)=0$ in order for existence to be a constant. As a non-member in the domain of $\mathrm{J}, \mathrm{B}$, existence as predicate adds no one thing to the manner in which $\mathrm{J}$ or $\mathrm{B}$ are articulated. 
We want to say of $\mathbf{J}, \mathrm{J}$ exists. However, if we take [3.31], we can negate this function and assert the identity of J. By doing so, we get by entering B, equivalent to NOT-J, we get:

\section{$[3.36] \mathrm{E}((\mathrm{NOT}-\mathrm{J})(\mathrm{E}(\mathrm{J})))$}

which is to say:

$\mathrm{E}($ NOT-J,J) or $\mathbf{J} \neq \mathbf{J}$. From [3.23] we see that without $\mathbf{J}$ there is no $\mathbf{B}$ for the same subject obtains different nominal functions. By [3.36] we see that presupposing negation before modality is inconsistent, for something could both exist and not exist simultaneously. All the while, implicit in what is negated is the object undergoing that operation.

To negate the existence of $\mathrm{J}$, taking existence as a predicate function of $\mathrm{J}$ whose result is just $\mathrm{J}$, presupposes that there is a $\mathrm{J}$. A predicate of existence adds nothing to our proposition or is straightforwardly contradictory. To suppose that we can predicate of $\mathrm{J}$ that it does not exist in one modality, that stipulation does not provide sufficient reason to assume that we cannot predicate its existence in another.

Modality, then, is found in the verb form of propositions; particularly between the verb 'is' and what $i s$, is or is not doing. We understand this as the application of the functional content of an assertion. Modality does not necessarily have to be stated unless to quantify what is by virtue of how what exists is categorized within a reference frame.8 Thus, conflating ontology with epistemology is revealed as false.

What are the consequences now that negation cannot precede modality? Treating modality as a predicate, we formalize that implicit in negation is the existence of the object negated. From our demonstration in [3.36], we can state:

\section{[3.4] IF (J THEN J) else (IF J THEN B)}

As a conditional's antecedent may or may not be true as long as the consequent is true, the antecedent of [3.4] states "IF (NOT-J OR J), THEN J". This becomes a description of the internal state of a possibility operator, a function as yet applied but wherever it is, that name is a possible constituent of the context it is used to construct. So represented, we illustrate the function of modality despite negation:

\section{[3.41] possibly-(IF J, THEN B)}

Negating that proposition we derive:

[3.42] NOT-(possibly-(IF J, THEN B), which results in necessarily-(NOT-B)

Assuming the axiom "IF NOT-B, THEN NOT-B", we obtain the same result as [3.42] gaining our possibility operator: NOT-necessarily-NOT-(B) equivalent to possibly(B). Modality survives negation and, better yet, negation is revealed as one of the operations derived from the function of modality. Negation, then, is one way in which modality is expressed, hence why modality precedes negation. In all, to negate is not to negate existence for only properties can be negated; it is to negate membership in the frame currently being considered.

This can be stated as a Baraka paradox: making existence a predicate or property definitive of the existence of the subject of that definition, without which there would be nothing to define, creates an infinite series of empty predicates or just leaves that subject as it is.9 In effect, this says no one thing at all. 
There is something of $\mathrm{X}$ that is " $\mathrm{X}$ " or just $\mathrm{X}$. Existence must precede negation and, thereby, cannot be a predicate. Hell is definitions.

To close this section, considering the following:

$[3.5]$. . be no---

$[3.51]$. . . no be---

According to the analysis above, [3.5] is valid and [3.51] is invalid. This demonstration provides an opening towards validating the consistency of Black forms of expression that were once stipulated as nonsense within a predetermined grammatical system. That grammar is now revealed as one imposed over that language made an object of a function constructing a racially categorical system. That system is external to the mechanism of creating and/or expressing Black thought. Within a system overdetermined by racial categorization, we find that blackness has a sense that takes care of its self.10

Two conclusions remain:11

[3.6] Origins are ontologically necessary but epistemologically contingent.

[3.61] Names are epistemologically necessary but ontologically contingent.12

In tandem with what will be evaluated below, identity as shown above is not a necessary component to the individual to which it is fixed, only to the reference frame employed. Names and their assertion index their function of application to the contexts in which it is appropriate to make that assertion because that context is a successor from the initial context in which that function was applied. Names, as identifiers, are only necessary to the frame of reference in which they are related to others as indicative of a system of categorization.

4. To propose a solution to this problem, consider the following. Our arguments thus far center around the tension between ontological and epistemological description due to a vagueness in the ability to refer to objects in the actual world.13 This is not a vagueness in language itself, understood as the mechanism for creating thought. However, in speaking of identity, it is my contention that the primary cause of the issue is a conflation between epistemology and ontology. Due to this confusion, only what is known or believed to exist, is assumed to be in the world. I say, what is known or believed not to exist, exists, but only as a non-entity in one reference frame out of many over what we consider to be the actual world. It is widely held that vagueness comes from semantic indeterminacy. We formalizes our argument in the following way.

Take an indefiniteness operator $\nabla$ which obtains an identity function between two objects. One represents the definiens or the definition $e$ composed of a finite disjunctive series of properties known by various physical and socio-historic features. The other is the definiedum or objective label $w$. We begin by stating that $e$ is indefinitely identical with $w$ :

\section{[4.1] $\nabla(e=w)$}

Next, we abstract a function that converts this indefinite identity statement into a form which predicates or expresses the property of being indefinitely identical to some other thing.

[4.12] $f_{\mathrm{x}} \nabla(x=w) e$ 
From this we can state that $\nabla(\mathrm{x}=\mathrm{w})$ is just a predicate function the likes of "W(. . )" which obtains or sets the role of or $e$ 's meaning within our reference frame once we exchange $e$ for $x$. Taking $e$ as its object within our state of affairs, $\mathrm{W}(e)$ expresses the concept "W"-ness. If $e$, an individual can obtain the function of "W" in this state. However, the result begets the truism that an object cannot be indefinitely identical to itself. So, to say $(x=w)$, we must assume that $w=w$. Therefore,

\section{[4.13] $\sim f_{\mathrm{x}} \nabla(x=w) w$}

This shows that one object has the property of definitely being itself - substitute the variable $x$ with $w$ and obtain $(w=w)$ - and the other object $e$ does not. $e$ is the object of the function W meaning $(e=w)$, thus $e$ and $w$ are supposedly interchangeable. The question becomes, do we have just $e$ or just $w$ under different names? Otherwise, we arrive at the contradiction that these two objects are the same object. In accordance with identity, objects that are identical must be interchangeable. They must have all the same properties. Therefore, if one or the other object has a distinct property, or we are able to discern between the two, then,

$$
[4.14] \sim(e=w)
$$

So, it is not the case that $e$ is identical to $w$ according to (4.14). If one object is indefinitely identical to the other, then those objects are not identical. Identity fails. However, this is not a straight contradiction. Identity is a relation between objects, which is not necessarily the solution of that function. These objects can be definitely not identical which logically defines the dual of our original operator. A names function of application is valid in so far as we know where it does not apply; if applicable everywhere, it's useless for its rules of application is determined to no one thing nor where. The dual, not-indefinitely identical, will be represented here as $\Delta$, to be understood as the operator "definitely."

$$
\text { [4.15] } \Delta \sim(e=w)
$$

which is read, it is definitely the case that $e$ is not identical with $w$. If we assume that definitely and indefinitely are converses, we derive,

$$
[4.16] \sim \nabla(e=w)
$$

The implication of this proof is that identity must not be vague, but can express, via the function of identity which is not the operation of interchangeability, some relation between objects expressing vague concepts. What is revealed is that an identifier predicated of an individual can only have functional content, exchange is not had between things but objects of thought. Changing our symbolism, we discover that if $w$ indefinitely identifies one of its constituents, say $e$, we can develop a predicate function such that " $x=E$ " which obtains as its object the label ' $w$ '. This has the effect of expressing a " $\mathrm{W}$ " reference frame.

[4.17] $f_{\mathrm{x}}\left(x=w\left(f_{\mathrm{y}}(y=e)\right)=\mathrm{W}(\mathrm{E}(.))\right.$.

If blindly following the interchangeability rule of identity, the possessor of this predicate, whose function or use gives meaning to the term $w$, must accept that, e.g. $w$ would not be "White" but some other thing; for "it is not the case that White=Europe" if White is a role utilized within a U.S. state of affairs. Its function stated as a predicate, ( $w=$ Europe), is empty. Europe itself is a predicate with no one object or nation, i.e. an empty sentence, e.g. $w=\mathrm{E}($ ?). The label White taken to be an object or individual is vague because, like an empty function or sentence without a subject, there is no way to clearly state which property obtains the function $(x=w) w$ qua $\mathrm{W}(. .$.$) . The functional predicate "W" remains indeterminate,$ which can formally be represented as W(?), and leaves us begging the question, "White" of what? 
Through the argument above, we disprove the notion of ontology being interchangeable with epistemology. The repetitive bifurcation of populations and their continuous division into subclasses so as to retain the starting classes position as the majority wreaks havoc on categorization. This is the case, even if, now with more and more constituents taken as evidence validating the state of affairs to be categorized, that starting class may actually be, by rule, the minority. We make sense of this in the following way.

Take the following demonstration. Starting from an individual $x$ such that $x$ affirms the category A, we have:

$4.2\{x: \mathrm{A}(x)\}$

From this, we can create a subset such that $x \neq x$, affirming what is NOT- $x$ but A alone.

$4.21\{\mathrm{~A}: x \neq x\}$ which is $\mathrm{A}(0), \mathrm{A}$ of no one thing.

NOT-A remains non-determinate. In order to discern or determine what NOT-A is, we define or re-label (4.2) as B,

$4.23\{x: \mathrm{B}(x)\}$ as the converse of $\mathrm{A}$.

Below we can show how this is at once a part of (4.2) and, yet, apart from it for it is included in the class (4.1). We continue this process, dividing (4.3) into

$4.24\{x: \mathrm{B}(x)\}$ and $\{\mathrm{B}: x \neq x\}=\mathrm{NOT}-\mathrm{A}$

where NOT-B is renamed C.

Proof of the general idea. If the set $\mathrm{x}$ is such by some property, that property in itself being such that $x \neq x$, for $\mathrm{A}=\mathrm{A}$ and $\mathrm{B}=\mathrm{B}$, unless $\mathrm{A}$ of $\mathrm{B}$ or $\mathrm{B}$ of $\mathrm{A}$, which would be a contradiction. As a subset of the other, $\mathrm{A}$ or B would entail that $x$ and $x$ 's negation would be in the same set. E.g. $\{x:\{\mathrm{B}: x \neq x\}$ and $\mathrm{A}(x)\}\}$. We must conclude that A and B are different, a relation between them is only constituted in a Null that is considered non-empty, i.e. no one thing, for null is the only class that has itself as a member. E.g. \{Null: $\{\mathrm{A}\}\{\mathrm{B}\}\}$; a relation stipulated between the two being a 1-1 ordering of the constituents of those sets within a domain that contains those sets as members. Here, the Null of each set becomes the zero of that function, for a function as yet applied expresses no one thing. Some function between A,B takes arguments in one set and pairs them with their respective solutions in the other. Thus, we can conclude one's relation to one's self is constructed out of Null, the content of that class qua category is functional when considered on its own. For $\{$ Null: $\{\mathrm{A}: x \neq x\}\{x: \mathrm{A}(x)\}\}$ making for the assertion of $\mathrm{A}$ the function of A's application due to its domain being defined by where it is inapplicable. Each successive assertion of " $\mathrm{A}$ " is a set of that set, for if $(x \neq x)=0$ and $f_{\mathrm{a}}=0=$ " $\mathrm{A}$ " as yet applied, then $\mathrm{A}=\{0\}$ which defines the domain of $f_{\mathrm{a}}$ as $\left\{f_{\mathrm{a}}\right\}=\mathrm{A}$, and A-successor $=\{\mathrm{A}: 0,1\}=\{0,1\}=\{0\{0\}\}$.

Referencing the above, if anything is a member of a "master" class, then it is either vacuous or determinate of no one thing in particular. We end up only deriving more and more negated classes: definitions of definitions or definitions which contain the term they are to define. This produces an infinite regress. As such, semantics are not primary but are expressed via the syntax or functional content of classifciation and their terms within a system of categorization. This process does have benefits. That which operates under NOT is no one thing and, therefore, is generative of any thing. The kernel to dismantling systems of control via categorization is implicit in that system's process of articulation. 
Just as well, systematic categorization is flawed as that which is not of the starting class becomes the basis for its very existence. The operation of categorization furthers itself through the accumulation of properties of benefit to that starting assumption by dispossessing them from other categories predetermined through encoded labels. These labels have no meaning save by becoming the objects of definitions externally, i.e. secondarily, imposed. In this case, what is considered a "master class," is the frame of reference itself (4.1).

With regard to the content of names being functional, for denotation taken as universally referring to a singular thing in the world fails, we find with the above that names, if they refer, identify, or denote the contexts of their appropriate application. The label "A" of the conditions in which $a$ obtains, is such that for every one of those elements, $a_{\mathrm{n}}$, A is not an element of $a$ for that name is not the object it names but the label taken for that object. Thus, if $a$ is a member of A, contradiction ensues. So, A is such that $a$ is an element of that class representing the context of proper application. Therefore, $a_{0}=\mathrm{A}$ and as such $a_{0}$ 's union with itself, constructing the set of appropriate contexts of asserting i.e. applying the function $\{a\}$ whose content is the zero of A. Thus, an is such that it cannot be proven in but labels some $a_{\mathrm{n}}$. Each successive assertion of "A" is appropriate with the union of a with that set thus ao is provable in Asuccessor and is the assertion of a 1 in that context, labeling the context prior " $A$ " according to Turing Ordinal logics.14 In accordance with the concept of separation, there were always be a subclass of a set which is not a member of that set. In this way, bo can be deduced from A as the label for that separate class the set of which is separate from the class from which it was derived. E.g. for the set of even numbers although made of a collection of natural numbers is not itself a natural number. A-ness is not a physical thing in the world, and yet its assertion enacts consequences because its content being functional, actively constructs the parameters in which it can be appropriately expressed as a function of the relation between the objects in its domain. 15

5. We will now apply the consequences of the above analyses to a cultural studies case. To analyze the unproblematic assertion of Black identity as Nothing in the reference frame of the Afro-Pessimist's paradigm, 16 let the usual constants of the propositional calculus be stated in arithmetical terms. Where conjunction is denoted by $\wedge$ or $\&$, it is now $\times$; disjunction by $\vee$ is + , defined as the disjoint union between entities: either or both; and negation by $\sim$ is - . The formation rules and truth-conditions for each remain unchanged. Using the truth conditions for entailment, we form the conditional in the following manner: it is not the case that there is a conjunction asserting that the antecedent be the case along with the negation of the consequent. This takes the place of $\supset$. The operator $=$ assigns a value to the class expressed by an assertion; it makes an identification where = is utilized to construct a formula it makes a statement about that formula's assertion, whether or not an individual, once translated for use in one reference frame from another, fits into, i.e. exists within, the system of classification of this new frame. Finally, to show membership to a class we utilize $\in$ such that an individual to the left of that constant is said to belong to the class to the right of that constant, e.g. $x \in \beta .17$ We follow the formation rules of logical propositions as usual. After translating formulae, we determine their value by arithmetic.

Taking on the Pessimist assertion, let 'Black' be $\beta$, 'White' be $\omega$ and 'absolutely nothing' be $\varnothing$, the class that is "Not." Not= "no one thing." Will see below the consequences of making an object identical to an operation without arguments. In the Pessimist's state of affairs one is identified as a member exclusively to Black or White. One is subject to the propositions of one categorization or another. 'Black' according to the Pessimist, suffers from a cultural death. Therefore, its value is 0 . The converse, 'White' is valued at 1. This paradigm is shown by the disjoint union of $\beta=0$ and $\omega=1$, ensuring that Black be Null and White a non-empty class asserted without ontologically over committing ones' self to more than what need be the case. Within the frame set up, this is valid for $1+0=1 ; 1$ marking validity by the definitions and parameters of this reference frame. From here, we can illustrate the Pessimist position as follows. 
There is an individual $x$ such that $x$ is a member of the product (conjunction) Black and Null classes,

[5.1] $x \in \beta \times x \in \emptyset=0$, for $x(\beta \times \emptyset)=0$

Therefore,

[5.12] $0 \times \varnothing=0$

However, utilizing the formation rules for $\supset$ (the negation of the conjunction of a true antecedent and false consequent), the Pessimist strictly defines Black Identity using a negative existential. Insofar as $\beta$ כ $\beta$ is $-(0 \times-(0))=0$, Pessimism defines White by what it is not:

[5.13] $-((x \in(\beta)) \times-(y \in \beta \times-((x=y) \times x \in \emptyset))=1$

translated, the above becomes,

$[5.14]-(0 \times-(0 \times-(0)))=1$

So,

$[5.15] 0=1 ?$

This is obviously not the case. We can assume that $y$ can also be a member of another category, namely $\omega$. Automatically, we see that an individual cannot be identical to a category, class, or set, only described as being a member or non-member of said category. Afro-Pessimism, therefore, is revealed as a reference frame over individuals; its negative existential over Black identity is only acquired through arbitrary stipulation; Pessimism's point of view is merely descriptive of, not interchangeable with, blackness itself.

Now, to challenge the reasoning of the Pessimist and come to our own conclusion. Let us posit the Pessimist's assertion as a conditional. "It is not the case that one can assert Black and negate its being Null," i.e. 'Black entails Not'.

$[5.2]-(\beta \times-(\varnothing))=0$

'White' entails not-'Black'

$[5.21]-(\omega \times-(0))=1$

but if $\omega=1$, then by our arithmetic,

$[5.23]-(1 \times-(0))=0$

Black's identity asserted, White's identity is negated. Just as well, in this state of affairs, the Pessimist paradigm being, by definition, the conjunction of White and Black individuals - for each individual there is what it is and what it is not-we can state,

[5.24] $1 \times 0=0$ 
Which can also be interpreted as White or not-'White',

$[5.25] 1+-1=0$

and, if one can either belong to the class White or Black,

$[5.26] 1+0=1$

We can derive,

$[5.27] 0=1$

Again, inconsistent. However, our reasoning reveals an interesting consequence. Within the AfroPessimist paradigm ' $0=1$ ' is inconsistent but within our lexicon, this formula allows for two interpretations. Either Black exists despite its negative existential or Black is the material condition for anything at all. It is possible utilizing our lexicon to show that one can derive 1 from 0 , some thing from no one thing. Take the following:

$[5.28] 0=1+-1$ i.e., $0=1-1$

The reduction of $1+-1$ to $1-1$ is unproblematic for here we see disjunction as the Boolean "and/or," which is inclusive. Both formulae have a solution of 0 which is the same as saying that it is true (1) that the proposition is false (0). To illustrate an exclusive disjunction would be to state, 1(1-1) which asserts that ' 1 ' is within the universe of discourse except ' 1 ' which becomes non-determinate or trivial. " 1 and non-1" or " 0 and non-0" merely expresses the limits of those respective categories, not what falls under the labels 1 or 0 . The question, then, is how many times can 1 and not-1, i.e. $1+-1$, be put into one to one correspondence with the members of the class ' $1+-1$ '? This operation asks how many subsets of our primary class's extension, i.e. the conjunction of all those subsets, are there included within that class, i.e. our primary class ' $1+-1$ '? Given one subsets to the exclusion of another within the extension of our primary class, how many times can we conjoin those exclusionary sets to match the extension of our primary class? (The arguments of this operation being individual but not mutually exclusive; therefore, creating or encompassing the limits of that world.) This operation can be called division. From this, it is easy to show in our arithmetic that,

[5.3] $0=\frac{(1+-1)}{(1+-1)}=1$

How many times does ' $1+-1$ 'enumerate' $1+-1$ '? 1 time, for anything divided by itself, just is 1 . It is possible to derive 1 from 0 , it is possible to form an expression of some thing, from no thing. Black, then, as 'absolutely nothing' is Everything. A universal categorization affirms the existence of no one thing. As every class has some thing which it is not, and that class being what is not to some thing else, the Null is the union of all classes - a part of and apart from each assertion. This harbors profound consequences for blackness considered as the Null, despite the inconsistency of Identity. Null entails and unifies All for the conditionals can be reduced to a negation operation: $\supset=-(\varnothing,-(\varnothing))$.

Blackness's modes of expression are not exhausted by categorization. Considering the relation above, $\omega$ over $\beta$ would mean $\frac{1}{0}$ which is the expression of infinity.18 Using the language in which the subject is overdetermined by strict identification, the subordinate can express a self out of and using what is no one thing in particular to the dominant regime of categorization which is indifferent to these expressions as they are not categorizable based on the identity framework employed. The Pessimist assertion, as well as 
that which asserts dominance, is inconsistent because blackness is indefinable outside of an, ultimately, arbitrary stipulation of Identity. Black identity, as distinct from Black subjectivity qua blackness as expressed by the function of the operation of the terms utilized to express the contexts in which it is a part, is epistemologically necessary within the Pessimist frame of reference, but ontologically contingent to category (class). This paradox follows when ontology and epistemology are conflated. Pessimism is brought to merely a point of view which is not definitive or exhaustive of blackness. Blackness, the subjectivity of those peoples, nondeterminate within this reference frame, has a circumference that is nowhere but a center found everywhere. To assert "Black=Null" affirms Black-ness as a function, an operation whose function expresses a way of being in the states of affairs in which it participates. The operation of that function, the expression of a form of life which, by necessity, is non-empty even if labeled as "nothing." As only a label, blackness outstrips any one attempt to make it just one thing; whereas its converse by definition must be affixed to an object so as to determine what it is not, the universal assertion of which inevitably leads to contradiction. 
NOTES:

1 LeRoi Jones (1964: The System of Dante's Hell, Grove Street Press)

2 see footnote below for implications when the expressions of blackness is made to populate the null class.

3 We say that $\mathrm{B}=b_{0}$ obtains where $\mathrm{a}$ is not an element of itself, i.e. the union of $\{a\}$ and $a 0$ represents the relation between $\mathrm{A}$ and null, thus is a function creating an ordered pair mapped into that space. From 0 we define a domain $\{0\}$ outside of which that function returns no one thing; and in that we get $\left\{a_{0}\left\{a_{\mathrm{n}}\right\}\right\}$. From there $\left\{a_{0}, b_{0}\left\{a_{\mathrm{n}}\right\}\left\{a_{0}, b\right\}\right\}=\left\{a_{0},\left\{a_{\mathrm{n}}\right\}, a_{0}, b_{0}\left\{a_{0}, b\right\}\right\}$ where $\mathrm{b}_{0}$ and a a are equivalent but not interchangeable and where $\mathrm{B}$ is related to A at it 0 thus $f(f(0))$ is A successor or just $f(0)$ alone and labeled B. We know that $b_{0}$ is not identical with any $a_{\mathrm{n}}$ even if $\mathrm{n}=1$ but we can abstract a function that relates 0,1 with an operation that can even make $0=1$ due to the presence of null in and in excess of each class (See [5]).

We see this from the following. A is from ao to an where $\mathrm{an}_{\mathrm{n}+1}=\mathrm{b}_{0}$ and $\mathrm{n}+1=f(\mathrm{n})$. So, $\{\mathrm{an}, \mathrm{b}\}$ is such that $\mathrm{A}$ and $\mathrm{B}$ are related insofar as $a<b$, starting from $a$ 's intersection with null. As bo is also a member of null, then where $a$ is not an element of $a$ is $b$ and is where $a_{0}$ is paired with and continues from $b_{0}$ on. In this way, every set constructed is embedded in null as null contains all functions of sets constructed zeros. A set then is a particular arrangement in null which may or may not intersect or disjointly unified, e.g. i) in null there is a gap between A,B; ii) A and B intersect when elements are coupled; or iii) There is no gap between A and B and A and B are not overlapping. Thus by (i) and (iii), A is closed relative some b element in B which is where $a$ is not- $a$, therefore null.

4 W.V.O. Quine (1961: "Reference and Modality” in From a Logical Point of View, Harper and Row).

5Alonzo Church (1941: "The Calculi of Lambda Conversion" in Annals of Mathematics Studies, Princeton University Press).

6 This follows lambda calculus rules. If $x$ is a variable then $x$ is an element of the domain defined by a function $f$. With $x$ as variable and $\mathrm{A}$ as an element of $\mathrm{F}$, then $f_{x}(\mathrm{~A})$ is an element of $\mathrm{F}$; if $\mathrm{A}, \mathrm{B}$ are elements of $\mathrm{F}$, then $(\mathrm{A}, \mathrm{B})$ is an element of $\mathrm{F}$ from here seen as a substitution instance such that: $(f(x(\mathrm{~A})) \mathrm{B})$ then $\mathrm{A}(x=\mathrm{B})$. Thus given two expressions A,B we read $f \mathrm{AB}$ as "if $f$ then A, else B."

7 E.g. Race purports a rule of application for which it itself does not have a rule to justify its own save for a previously held belief. It is not necessary for an individual to be categorized or racially identified in order for them to exist in the world. Racism begets Race, the operation of its application expresses the concept whose only content is the function that constructs the contexts in which these stipulations are asserted. If racism is expressed by the implementation of racial categories, thus is the function of the application of those categories themselves, a category with no objects only contains the function that collects what is appropriate and as yet applied. The zero of a function is the category itself. "If Race then racism" is equivalent to "if R then 0," which states "if Race then false." Proof in [1]. Taken this way, "if Race then racism," commits the traditional logical fallacy of affirming a consequent: "if Race then racism"; racism, therefore Race. Assuming Race as a given leaves open the question, how did Race get there in the first place?; "if racism then Race," Race therefore racism shows the logical fallacy inherent in racism; and taking the valid inference if racism then Race, racism, therefore Race exhibits how Racial categories are constructed, how credence is afforded them, and explains the mechanism behind the social organization we inhabit. The property of "Black"-ness expressed via various functions of the label "Black" only gains significance by how that label is used within this context. Black identity, then, is the result of no one feature alone; blackness can express itself with any one feature and, therefore destroys systems of controlled existence by virtue of a racialized label.

8 F.P. Ramsey (1925: "Universals" in Mind, 34, 136, pp. 401-417) states this is found in the various verb forms of 'to be'. Interestingly enough, LeRoi Jones/Amiri Baraka (1963: Blues People, William Morrow and Company) and Zora Neale Hurston (1934: Characteristics of Negro Expression) came to a similar conclusion. 
9 This is a rather precarious result when one takes on an Afro-Pessimist position: blackness as a zone of non-being; a site of cultural death; Black is/as NOT. See Jared Sexton (2011: "The Social Life of Social Death: On AfroPessimism and Black Optimism" in InTensions Fall/Winter: 5), Orlando Patterson (1982: Slavery and Social Death, Harvard University Press), Frank Wilderson III (2008: Incognegro: A Memoir of Exile and Apartheid, South End Press), et. al.

10 See Ludwig Wittgenstein (1921: Tractatus Logico-Philosophicus, Kegan Paul, Trench, Trubner \& Co., LTD London; New York: Harcourt, Brace \& Company, Inc.) and Richard A. Jones (2013: The Black Book: Wittgenstein and Race, University Press of America).

11 Take the 2018 redefinition of Race in the U.S. Census as an example. The Census Bureau revised the formula expressing racial categorization. The matrix of categories constituting the reference frame of the Census are: White, Black/African-American, American Indian/Alaskan Native, Asian, and Native Hawaiian/Other Pacific Islander. It is well documented that the socio-political apparatus organizing the U.S., mapped over the indigenous peoples and landmass of the Americas, is based on a Black-White continuum at the expense of the very populations it sought to quantify - cf. historical construction of racial categories since the first U.S. Census. Subcategories are devised from these two categories. One could say that these subcategories are devised out of Black alone, as White is the nominative assumption with everything else being not-White. (cf. the definition of White as other than any other category.)

Our terms are, in accordance with the Census, as follows: (w)hite: A person having origins in any of the original peoples of Europe, the Middle East, or North Africa; (b)lack/African-American: A person having origins in any of the Black racial groups of Africa, i.e. African-American and Black are made interchangeable: "Black"=Black origins.

Thus,

$$
[1] \mathrm{w}=\{\text { Europe, Middle East, North Africa }\} \quad[2] \mathrm{b}=\{(\mathrm{b}) \text { lack }\}
$$

If $b$ is equivalent to African-American, our first issue arises from within the category labeled "w" itself. In $w$, we have a feature indicative of $b$-ness, "African-..." included in the property "North Africa" of $w$. The same feature appears in both descriptions. To obtain the label "Black," one must assume the subset of features encompassed by the property "Africa". We assume North Africa is a part of the so named "African" continent and yet, here stands a part from it as the former may obtain the label "White" as well.

"Black" peoples can be found in the "origins" made definiens of White and other categories. Due to slavery, as well as trans-Atlantic and Pacific travel before and after that period, the Census admits "racial mixture" by checking multiple boxes on the survey and that people of "Hispanic, Latino, or Spanish" identity may be of any race but remaining not-White. Just as well, it is curious that 'Middle East' is claimed under "White" categorization, an area characterized as being not-White.

The formula $f_{x}(x=b)$ creates a predicate whose content is " $(\mathrm{x}=\mathrm{b})$ ". Substituting the variable $x$ with $b$ renders $b=b$, equivalent to the predicate $\mathrm{B}$ obtaining the function $b$ as an object. There is no ambiguity, just affirmation of functional content. Black remains non-determinate as there is no one function it obtains that locks it into a single context. The functional content of $b$ can obtain any role dependent on the frame of reference in which a predicate can be formed with which (b)lack-ness can be expressed. $b$-ness' expression, then, is appropriate to reference frame but not caused by that context, nation, etc.

"White"-ness, then, is contradictory if considered an object within its own frame of reference. $w$ is not strictly interchangeable with its set of defined origins. This inconsistency is exhibited when considering: $f_{x}(x=w)$ as the functional content of the predicate $\mathrm{W}$, with input $e$, then $w=e$, but if $w=w$ and $w=e$, then $w$ cannot be $w$. How can "White"-ness be both an object of the Census but defined by all that it is not? As such, "White" cannot be an object in the state it organizes for it becomes so narrowly defined with the addition of more and more categories limiting access to that category, that no one can inhabit it. If membership is to be subordinate to that definition as dominant, to be both is contradictory. The frustration of that predicament is taken out on those defined as not. W's content as category is merely functional. It contradicts itself when asserted for each term in its definition has functional content 
as well: if any one of those functions obtain, a contradiction; if nothing as yet, it's vacuous. As a "function" of functions it is revealed as a mechanism of control, organizing a state of affairs despite what is there. As for $b$, this result does not imply $b$-ness is one and only one thing, but that it is no one thing but a function whose application constructs the contexts in which $b$-ness obtains. This is not dependent on any one thing, but as a function, its operation expresses $b$ in ways appropriate to context, possibly novel in alternative contexts, but not necessarily caused by the context in which it was previously a member. As such, and due to our proof in [1], we know by virtue of the very terms of this system, $b$ will always be non-empty, populated by all that is negated from $w$, that negation affirming what is and was already there in order for the assertion of $\mathrm{W}$ to occur in the first place. $b$ is always in excess of what $w$ stipulates of it. W's assertion begs the question, which thing does it denote and thus how it can maintain its being one thing and yet some other? Is it that the label White is vague? Or, is it the list of properties which define it? See United States Census Bureau (2017: "Research to Improve Data on Race and Ethnicity"; and 2018: "About Race").

12 Saul Kripke (1980: Naming and Necessity, Harvard University Press).

13 Gareth Evans (1978: “Can There Be Vague Objects,” Analysis, Volume 38, Issue 4).

14 Alan Turing (1938: Systems of Logic Based on Ordinals).

15 This process is done until the features of those cultural products are no longer useful-i.e. appropriation is defined as the incorporation qua dispossession of cultural expression from one context for objective use in and to the benefit those outside of that "originating" context. This is stated in the U.S. Census itself which denies the, "attempt to define race ... anthropologically. . ." However, as displayed above, the use of "origin" in these definitions evinces the epistemological basis for the ontology enforced in this document. The disavowal of this term used within the document is evidence of a belief system which is used to quantify populations, quantification being a basis for epistemology now utilized to substantiate the racial ontology of the U.S. Ideology as a belief in a belief system allows those who wield it to eschew accountability which openly admits that system's flaws.

16 See: Wilderson III, Sexton, and Patterson.

17 I owe much to P.F. Strawson (1952: Introduction to Logical Theory, Wiley \& Sons New York), for the translation of these logical constants.

18 See Victor Peterson II (2018: Raising Pure Hell: a General Theory of Articulation, the Syntax of Structural Overdetermination, and the Sound of Social Movements, London: King's College) and Denise Ferreira da Silva (2017: "1 (life) $\div 0$ (blackness) $=\infty-\infty$ or $\infty / \infty$ : On Matter Beyond the Equation of Value." in e-flux journal, Issue 79). 